\title{
A Novel Pressure Film Approach for Determining the Force Imparted by Clear Removable Thermoplastic Appliances
}

\author{
Laura J. Barbagallo, ${ }^{1}$ Gang Shen, ${ }^{1}$ Allan S. Jones, ${ }^{2}$ Michael V. Swain, ${ }^{3}$ Peter Petocz, ${ }^{4}$ \\ and M. Ali Darendeliler ${ }^{1}$ \\ ${ }^{1}$ Department of Orthodontics, Faculty of Dentistry, Sydney Dental Hospital, The University of Sydney, Level 2, 2 Chalmers \\ Street, Surry Hills, NSW 2010, Australia; ${ }^{2}$ Electron Microscope Unit, The University of Sydney, Surry Hills, Australia; \\ ${ }^{3}$ Biomaterials, Faculty of Dentistry, The University of Sydney, Surry Hills, Australia; and ${ }^{4}$ Department of Statistics, Macquarie \\ University, Sydney, Australia
}

(Received 15 December 2005; accepted 6 December 2007; published online 18 December 2007)

\begin{abstract}
The force imparted by removable thermoplastic appliances (RTA) onto teeth has not been investigated in the past. This investigation was designed to explore a novel methodology to measure the magnitude and identify the pattern of this force. Eight patients with moderate malocclusion were selected. In each patient, the palatally mal-positioned upper first premolar was corrected by wearing a series of four ClearSmile ${ }^{\circledR}$ RTA over a duration of 8 weeks. When constructing RTA, the ClearSmile ${ }^{\circledR}$ Company was advised that the amount of movement to be programmed into each appliance was $0.5 \mathrm{~mm}$. The Pressurex ${ }^{\circledR}$ film was used to measure the pressure generated by ClearSmile ${ }^{\circledR}$ RTA against the palatal surface of the upper first premolar for buccal tipping movement. Three measurements were conducted respectively upon the issue and retrieval of each appliance (after 2 weeks of wear), resulting in 24 pressure measurements for each patient. Digital imaging and spectrophotometry analysis were employed to quantify the stain intensity mounted by the pressure on the films. The irrelevant forces were subtracted out to allow an assessment of the force purely acting to buccally repositioning the tooth. The results revealed that (1) the mean force magnitude over 2 weeks of RTA wear was $1.12 \mathrm{~N}(\mathrm{SE}=0.72 \mathrm{~N})$; (2) the higher force magnitude of $5.12 \mathrm{~N}(\mathrm{SE}=0.80 \mathrm{~N})$ seen at the issue of the appliance declined drastically to $-2.67 \mathrm{~N}$ at the time of retrieval. These findings suggest that ClearSmile ${ }^{\circledR}$ RTA exerts a high level of force against the tooth to be moved at the initial stage followed by a rapid force diminish.
\end{abstract}

Keywords-Pressurex film, Pressure measurement, Removable thermoplastic appliances, ClearSmile, Spectrophotometry.

\section{INTRODUCTION}

Since Kesling in orthodontics first introduced the use of a flexible removable orthodontic appliance for

Address correspondence to M. Ali Darendeliler, Department of Orthodontics, Faculty of Dentistry, Sydney Dental Hospital, The University of Sydney, Level 2, 2 Chalmers Street, Surry Hills, NSW 2010, Australia. Electronic mail: adarende@mail.usyd.edu.au minor tooth movements following fixed appliance therapy in $1945,{ }^{8}$ as an alternative to fixed appliances treatment, the moderately mal-positioned teeth could be corrected by use of a series of consistent removable thermoplastic appliances (RTA) that reposition the teeth in a gradual, sequential, and consecutive way. $^{2}$ Two commercial RTA systems are now available in Australia: ClearSmile ${ }^{\circledR}$ and Invisalign ${ }^{\circledR}$. ClearSmile ${ }^{\circledR}$ has been using thermoplastic "invisible appliances" to treat more complex malocclusions than those proposed by previous authors such as Sheridan. ${ }^{17}$ From a cast of a polyvinyl-siloxane (PVS) impression, the ClearSmile ${ }^{\circledR}$ technicians manually reset teeth in sequential stages on the plaster model and construct a series of thermoplastic appliances. The appliances are constructed from Erkudor ${ }^{\circledR}$ (polyurethane) thermoplastic blanks of $0.8 \mathrm{~mm}$ thickness.

No reports can be found in the orthodontic literature that measure the force pressure imparted by RTA onto teeth. In the past, strain gauge based systems were commonly used in the field of biomechanical forces measurement as this was a method that offered reliable results. ${ }^{14}$ If the modulus of elasticity of the material under strain is known, the pressure that it is applying can be calculated from its strain data. There are, however, a number of shortcomings associated with the use of strain gauge systems. The gauge must be bonded to the test material, leading to the scenario where the contact and the chemical reaction associated with adhesion may alter the materials inherent flexibility. ${ }^{18}$ The irregular shape of the appliance surface leads to difficulties with uniformity of bonding. The data provided from each strain gauge is related only to its discrete position, thus there is the potential to miss areas of high pressure or high pressure-gradients. When used in a moist environment, waterproofing 
is required to be applied to the circuitry, which also may alter the flexibility of the test material. ${ }^{18}$

The pressure transducers, which are applied between two surfaces, are another popular alternative method of pressure measurement. ${ }^{11,20}$ However, the introduction of this additional transducer layer between the two areas of contact will result in a greater pressure reading than the actual situation, unless one of the surfaces is implanted with the transducer. The process of implantation disrupts the properties of one of the contact surfaces. ${ }^{13}$ Some pressure transducers have the advantage of producing "real-time" data which is readily interpreted. ${ }^{1}$ However, they also share many of the same limitations as strain gauges including the limited ability to only measure pressure in a discrete location, high cost, and the need for electrical circuitry which must be waterproofed. ${ }^{6,11}$

To avoid interfering with the strain that is being measured, technologies such as pressure sensitive films are being developed that endeavor to reduce the thickness of the pressure sensor in order to minimize the interface contact. Pressurex ${ }^{\circledR}$ (Fuji Photo Film Co., Ltd Tokyo, Japan), a pressure sensitive polyester film, provides direct pressure distribution and quantification means. The film is usually supplied in two sheets: A-transfer and C-developer films. The combined width of the two films is $90 \pm 5 \mu \mathrm{m}$. When pressure is applied to the sheets, fluids are released from microscopic bubbles on the surface of the A-film. The fluids react with an active layer on the surface of the C-film to produce a pink stain. The optical density response of the C-film is demonstrated by the change in stain intensity from pale pink to dark red. ${ }^{9}$ It has been reported that C-film stain intensity increases in a fifthorder relationship with an increasing applied pressure. ${ }^{9}$ Hale and Brown ${ }^{5}$ state that a nonlinear correlation exists between optical density and contact stress magnitude where a cubic polynomial relationship is found. The differing calibration curves found in different studies may indicate that the calibration correlation is affected by the data collection method and the nature of two-surface interface.

In this study, the Pressure ${ }^{\circledR}$ film was placed underneath thermoplastic appliances resulting in pressure stain samples. The color density of stained films may then be compared with stain samples of known pressures to determine the pressure imparted by the clear appliances. A combination of digital imaging and spectrophotometry facilitate this comparative procedure.

Therefore, by the use of Pressure ${ }^{\circledR}$ film, the aims of this study were (1) To determine the magnitude and the pattern of the force generated by ClearSmile ${ }^{\circledR}$ RTA to displace the mal-positioned tooth, and (2) To test the feasibility and reliability of a film approach in examining force and pressure.

\section{MATERIALS AND METHODS}

\section{Patients and Clinical Procedure}

Eight subjects were selected from the patients participating in the ClearSmile ${ }^{\circledR}$ RTA orthodontic treatment (Central Sydney Area Health Service Ethics Review Committee, Ethics approval No. X03-0224). The patients presented with palatally mal-positioned upper first premolars. In each patient, the palatally mal-positioned upper first premolar was corrected by wearing a series of four ClearSmile ${ }^{\circledR}$ RTAs over a duration of 8 weeks, with one new RTA for every 2 weeks. When constructing RTA, the ClearSmile ${ }^{\circledR}$ Company was advised that the amount of movement to be programmed into each appliance was $0.5 \mathrm{~mm}$.

The two Pressurex films (A- and C-film) (Fuji Photo Film Co., Ltd Tokyo, Japan) were cut with a scalpel to the approximate size of the palatal surface of the first premolar. A piece of adhesive tape was attached to the buccal and palatal edges of the two films. Adhesive tape was also used to temporarily adhere the film to the RTA such that the two pieces of Pressurex ${ }^{\circledR}$ film sat inside the palatal surface of the RTA. The RTA with the film and tape in place was seated in the patient' dentition, with all of the buccally directed force that was applied to the palatal surface of the tooth would consequently be transmitted and therefore recorded on the film (Fig. 1). After a 15-s of interval, the RTA was removed. The regions where the adhesive tape was attached to the film were cut off with a scalpel and the tape and sliver of film discarded. Three of these film measurements were conducted, resulted in a series of stained films (Fig. 2). This process was carried out at the time of issue of new RTA and the time for the end

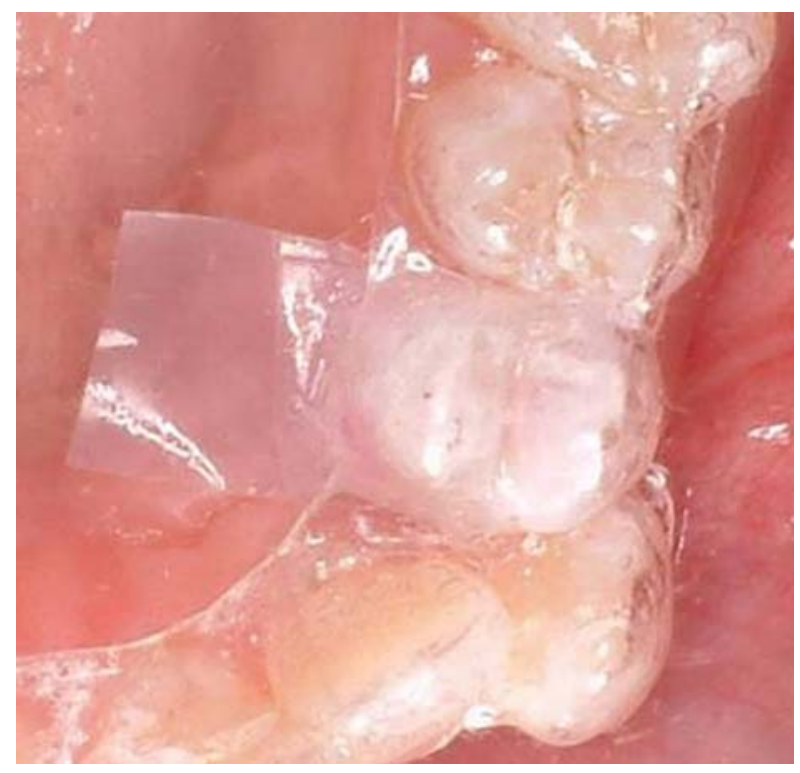

FIGURE 1. Pressure measurement with Pressurex ${ }^{\circledR}$ film. 


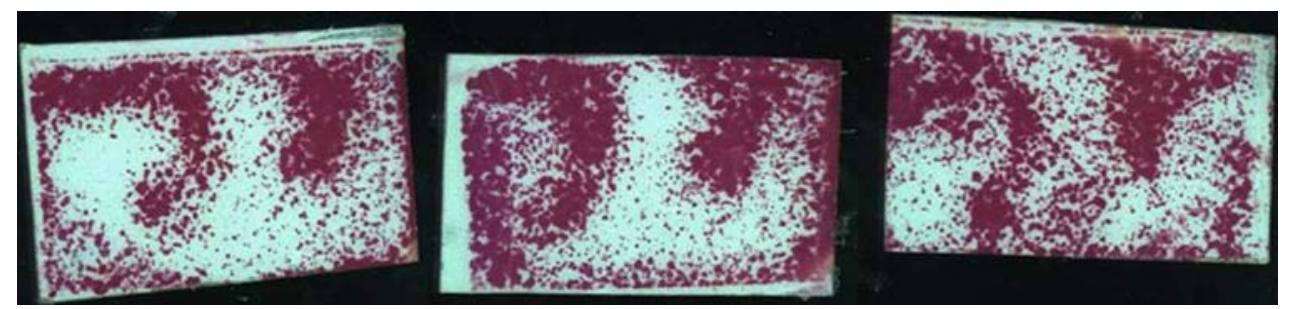

FIGURE 2. An example of a series of three pressure measurements from the palatal surface of the first premolar made from the same ClearSmile ${ }^{\circledR}$ RTA.

of wear of this RTA, spanning 2 weeks. As each patient received four RTAs with consecutive 8 weeks, 24 pressure measurements were conducted per patient.

\section{RTA Force Assessment with Pressurex Films}

\section{Calibrating the Pressure Against the Stain Intensity}

To establish the mathematic relation between degree of force and that of the stain incurred to the films, a pilot study was conducted where the known pressures were applied to the Pressurex ${ }^{\circledR}$ film. A cup attached to a rod with a rounded end was employed to apply weight to a disc with a known radius and thus a known contact area. The cup and rod were placed in a sliding apparatus. Known weights were placed in the cup and thus the rod applied this weight via the disc onto the Pressure ${ }^{\circledR}$ film. The known weight (including the cup, its contents, the rod and disc) was applied to the Pressure ${ }^{\circledR}$ film for $15 \mathrm{~s}$ (Fig. 3). As the nature and duration of two-surface interface (disc versus the film) in the calibrating pilot study were similar to that (RTA vs. the film) in the main study, there was a consistency in load rate between the calibration stain generation and the collection of the test data.

\section{Excluding the Irrelevant Forces}

When the RTA was seated into the dental arch, the generation of some forces that were not contributing to the buccally tooth movement occurred, leading to a falsely increased reading from the stained films. These unavoidable additional forces were caused, namely, by the vertical component of the force when seating the appliance and the inherent thickness of the Pressurex ${ }^{\circledR}$ A- and C-film combinations. To estimate and then to subtract these irrelevant forces, the following measurements were performed. A passive RTA designed to produce no tooth movement was constructed on the study models of the two patients. Six pressure film readings were conducted for each passive RTA. These pressure stains were the results of all influences except for tooth movement pressure and would hereon be referred to as the "passive RTA pressure." On another patient study model, the A- and C-pressure films were temporarily bonded to the region of interest and a passive RTA was constructed to fit over this combined film layer. The temporarily bonded film was then discarded. The resultant RTA had a region of "relief" which was equal to the film thickness. Thus when the pressure of this RTA was measured, by placing a new film in the region of relief, the measured pressure could be assumed to be caused by seating and shearing (with no pressure added by the film thickness). This measured pressure would hereon be referred to as the "relief pressure."

\section{Digital Imaging and Spectrophotometry Analysis}

A light microscope (SZ-FO Dissecting Microscope, Olympus, Japan) linked to a digital camera (Color View
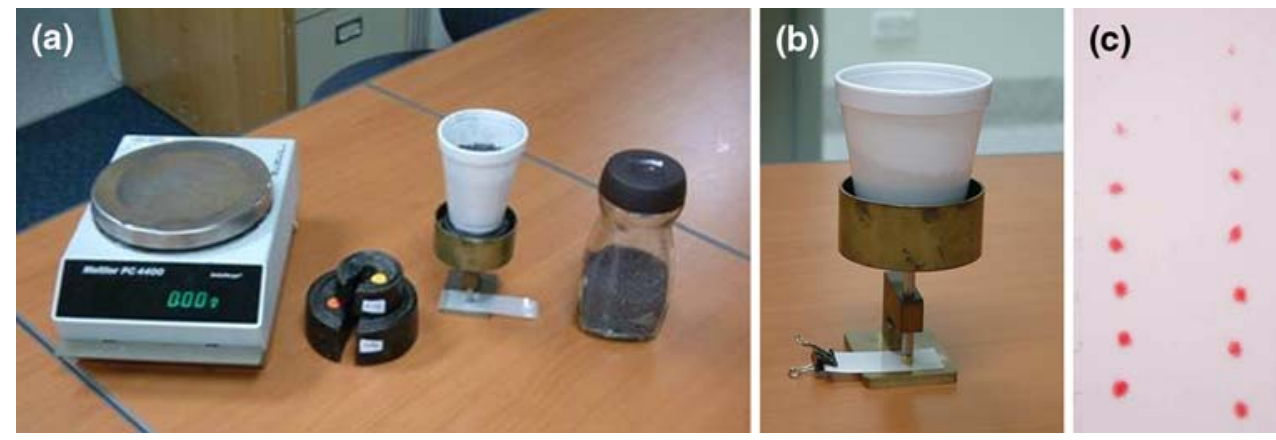

FIGURE 3. Procedure for application of known pressures to the Pressurex ${ }^{\circledR}$ film. (a) A digital scale (Mettler PC 4400, Delta Range), lead bearings, and the cup and rod in the sliding apparatus applying pressure to the Pressurex ${ }^{\circledR}$ film. (b) A closer view of the cup and rod sliding apparatus, disc and the Pressurex ${ }^{\mathbb{B}}$ film. (c) A series of known pressure application samples. 
Soft Imaging System, Olympus, Japan) facilitated digital image capture of all of the films. A separate image was taken of each film. AnalySIS Soft Image System software (GmbH, Johann-Krame-Weg 39, D-48149 Munster, Germany) was utilized to measure the area of each pressure stain according to a color intensity threshold. The color intensity threshold remained consistent for the duration of the investigation.

The pink dye produced on the C-film was found to be highly soluble in acid-alcohol $(70 \%$ ethanol, $1 \% \mathrm{HCl})$. Acid-alcohol elution was individually conducted with the clinical and known pressure films, each in a volume of $200 \mu \mathrm{L}$ (measured with a micro-epindorph pipette). The individual elutions were each agitated with a vortex mixer for $5 \mathrm{~min}$, which allowed all of the dye on the film to be eluted into solution. A test was conducted to measure the effectiveness of elution. Five C-films were removed from the elution solutions and re-eluted in separate fresh $200 \mu \mathrm{L}$ acid-alcohol solutions. The re-elutions were found to have no dye concentration (the same absorption peak as acid-alcohol).

Spectrophotometry was employed to determine the concentration of dye of the eluted pressure-stained C-films with the Cary 5E UV-Vis-NIR Spectrophotometer (Varian Australia Pty Ltd.). The measured absorption at $496 \mathrm{~nm}$ (the peak of the absorption spectrum for the pink dye) was directly proportional to the dye concentration. This is in accordance with the Beer-Lambert Law, provided that the optical path length of the solution and the radiation wavelength is kept constant. ${ }^{15}$ The known pressure films' elutions were analyzed with the spectrophotometer to determine the dye concentrations that corresponded to each pressure. Thus a known pressure versus concentration calibration curve was constructed. The concentrations of the clinical pressure films elutions were also determined with the spectrophotometer. By relating the clinical film concentrations to the calibration curve and its regression formula, the pressure measured by these films was thus calculated.

\section{RESULTS}

\section{Calibrated Spectrophotometric Concentration Against Known Pressure}

The calibration curve constructed from the spectrophotometry absorption data for the known pressure films was linear (Fig. 4). The regression equation calculated from the known data was:

$$
\text { Pressure }=0.766+13.15 \times \text { Absorption }
$$

(where $R^{2}=0.98, S=0.33$. The $p$ value for the regression was less than 0.001 ).

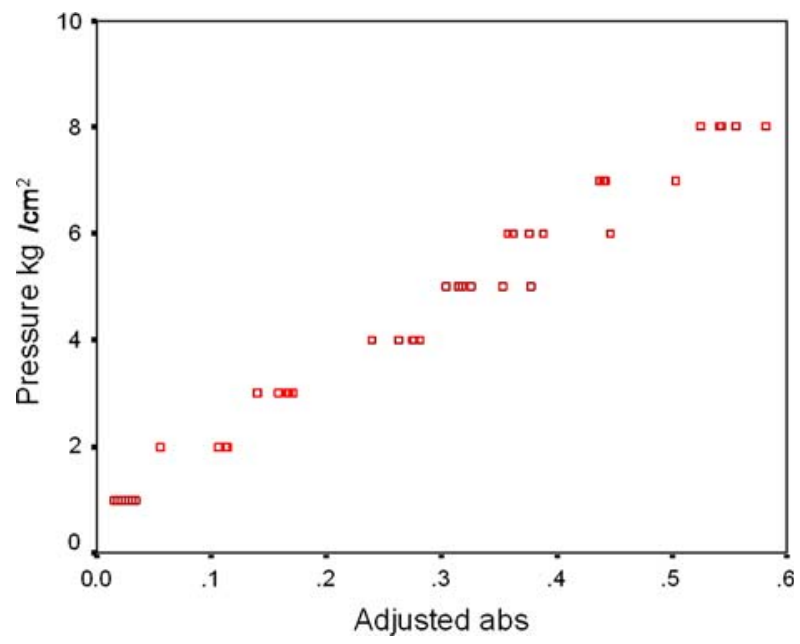

FIGURE 4. Calibration curve constructed from the absorption data for the known pressure films. Regression formula: Pressure $=0.766+13.15 \times$ Absorption, where $R^{2}=0.98$, $S=0.33$.

This regression formula and calibration curve were then used to determine the pressure from the individual patients' data.

\section{Force Magnitude Exerted by RTA}

As mentioned earlier, the area for each observation was measured with the light microscope-digital imaging system. Once the pressure $\left(\mathrm{N} / \mathrm{mm}^{2}\right)$ and area $\left(\mathrm{mm}^{2}\right)$ values were determined, the force imparted upon each pressure film sample was thus calculated via the formula:

$$
\text { Force }=\text { Pressure } \times \text { Area }
$$

The force imparted by the ClearSmile ${ }^{\circledR}$ RTA onto the palatal surface of the patients' first premolars was calculated from the Pressure ${ }^{\circledR}$ film pressure and area measurements. This mean force was found to be $24.19 \mathrm{~N}(\mathrm{SE}=0.44 \mathrm{~N})$. This calculation included the force imparted by the RTA, plus the unavoidable irrelevant pressures (Table 1). The mean force measurements for each of the eight individual patients are shown in Table 2.

TABLE 1. Mean force imparted by the ClearSmile ${ }^{\circledR}$ clinical, passive and relief RTAs.

\begin{tabular}{lrrrrrr}
\hline $\begin{array}{l}\text { Type of } \\
\text { data }\end{array}$ & Mean & $N$ & SD & $\begin{array}{l}\text { SE of } \\
\text { Mean }\end{array}$ & Minimum & Maximum \\
\hline 1. Clinical & 24.19 & 202 & 6.25 & 0.43 & 11.61 & 45.05 \\
2. Passive & 22.96 & 12 & 1.97 & 0.56 & 20.18 & 27.10 \\
3. Relief & 11.39 & 6 & 2.00 & 0.82 & 9.72 & 14.81 \\
\hline
\end{tabular}

Predicted force: N (unit). 
TABLE 2. Mean of the forces imparted by the four Clear Smile ${ }^{\circledR}$ RTAs for the eight individual patients.

\begin{tabular}{lcccc}
\hline & & & \multicolumn{2}{c}{$95 \%$ Confidence interval } \\
\cline { 4 - 5 } Subj. & Mean & SE & Lower bound & Upper bound \\
\hline 1 & 21.67 & 0.89 & 19.91 & 23.43 \\
2 & 25.43 & 0.87 & 23.71 & 27.15 \\
3 & 26.81 & 0.89 & 25.05 & 28.57 \\
4 & 21.16 & 0.89 & 19.40 & 22.91 \\
5 & 22.33 & 0.87 & 20.60 & 24.05 \\
6 & 21.49 & 1.02 & 19.46 & 23.52 \\
7 & 25.68 & 0.89 & 23.92 & 27.43 \\
8 & 26.79 & 0.70 & 25.39 & 28.19 \\
\hline
\end{tabular}

Dependent variable: Predicted force: N (unit).

TABLE 3. Mean initial and mean final force imparted by the ClearSmile $^{\circledR}$ RTA (for the eight patients).

\begin{tabular}{lcccccc}
\hline Time & Mean & $N$ & SD & SE of mean & Minimum & Maximum \\
\hline 1. Start & 28.09 & 101 & 5.64 & 0.56 & 17.96 & 45.05 \\
2. End & 20.29 & 101 & 3.99 & 0.39 & 11.61 & 28.67 \\
Total & 24.19 & 202 & 6.25 & 0.43 & 11.61 & 45.05 \\
\hline
\end{tabular}

Predicted force: $\mathrm{N}$ (unit).

The mean initial force upon RTA issue was $28.09 \mathrm{~N}$ $(\mathrm{SE}=0.56 \mathrm{~N})$, and the mean final force upon RTA retrieval was $20.30 \mathrm{~N}(\mathrm{SE}=0.40 \mathrm{~N})($ Table 3$)$.

As to the irrelevant forces, the mean force imparted by the passive RTA due to film thickness, instantaneous seating and shear pressure was found to be $22.97 \mathrm{~N}(\mathrm{SE}=0.56 \mathrm{~N})$ (Table 1). The mean force imparted by the relief pressure was found to be $11.39 \mathrm{~N}(\mathrm{SE}=0.82 \mathrm{~N})($ Table 1$)$.

After subtracting all the irrelevant forces, the mean initial force at issue of the RTA was $5.12 \mathrm{~N}$ $(\mathrm{SE}=0.80 \mathrm{~N})$ and the mean force on RTA retrieval was a negative $-2.67 \mathrm{~N}(\mathrm{SE}=0.69 \mathrm{~N})$. Thus the mean force exerted by a ClearSmile ${ }^{\circledR}$ RTA over the 2-week period of application onto a first upper premolar for buccal tipping movement, after subtracting all of the irrelevant pressure sources, was $1.12 \mathrm{~N}$ (SE $=0.72$ $\mathrm{N})$. This sum was calculated assuming that the RTA forces between two time points changed in a linear pattern.

Since the mean initial force was 4 times greater than the mean force over the 2 weeks, it was not likely that there was a linear force change; it was more likely that it declined rapidly as the material deteriorated.

\section{DISCUSSION}

The force magnitude and pattern exerted by RTAs onto mal-positioned teeth has not been investigated before. In this study, the force imparted by the ClearSmile ${ }^{\circledR}$ RTAs onto the palatal surface of the patients' first premolars to produce buccal movement was quantified by utilizing Pressurex ${ }^{\circledR}$ film integrated with digital imaging and spectrophotometry analyses. Spectrophotometry is the quantitative measurement of the reflection or transmission properties of a material as a function of wavelength. The instrument operates by passing a beam of light through a sample and measuring the intensity of light reaching a detector. This method has been proven to be accurate, precise and reproducible while being simple, cheap and less time consuming. ${ }^{19}$

While the film is moisture tolerant, the presence of bodily or cadaveric fluids can interfere with the transfer of stain material from the A-film to the C-film. This has led to the sealing of film to prevent this issue as did in some studies. ${ }^{10,12}$ In this study, the tooth to be tested was air dried from saliva before the films together with RTA were inserted. As the test duration lasted for only $15 \mathrm{~s}$, the films were therefore well kept from oral moisture.

The magnitude of the mean force, when unadjusted for passive appliance measurement, is substantially higher than would be anticipated in the clinical setting (Tables 1 and 2). A number of reasons for these high measurements recorded with the film have been suggested: (1) The pressure film records an overall force which may be resolved into a vertical as well as a horizontal component, which is clearly illustrated by a vector diagram (Fig. 5). As shown in Fig. 5, around the cusp tips the vertical component force $\left(F_{\mathrm{v}}\right)$ is quite pronounced, whereas the corresponding horizontal component force $\left(F_{\mathrm{h}}\right)$ at this location is diminished. This large vertical component significantly adds to the overall derived pressure value, but does not contribute to the buccally tooth movement, which is solely achieved by the horizontal component. This will therefore tend to result in a high-pressure stain record and a correspondingly high force value being calculated. In orthodontic practice, both the conventional wire appliances and the removable appliances do not have a large vertical force component, therefore the majority of the force is imparted in a horizontal direction resulting in tooth movement. ${ }^{3}$ (2) The sliding contact of tooth and appliance surfaces produces a shear effect. During seating, the drag of undercut component of the appliance creates a transient pressure that is recorded by the film. (3) The inherent thickness of the Pressurex ${ }^{\circledR}$ A- and C-film combinations $(90 \pm 5 \mu \mathrm{m})$ is expected to produce a greater pressure onto the tooth than if this extra layer was absent.

The analysis of the initial force $(5.12 \mathrm{~N})$ upon RTA issue and final force $(-2.67 \mathrm{~N})$ upon retrieval was conducted and the calculated mean force over 2 weeks 


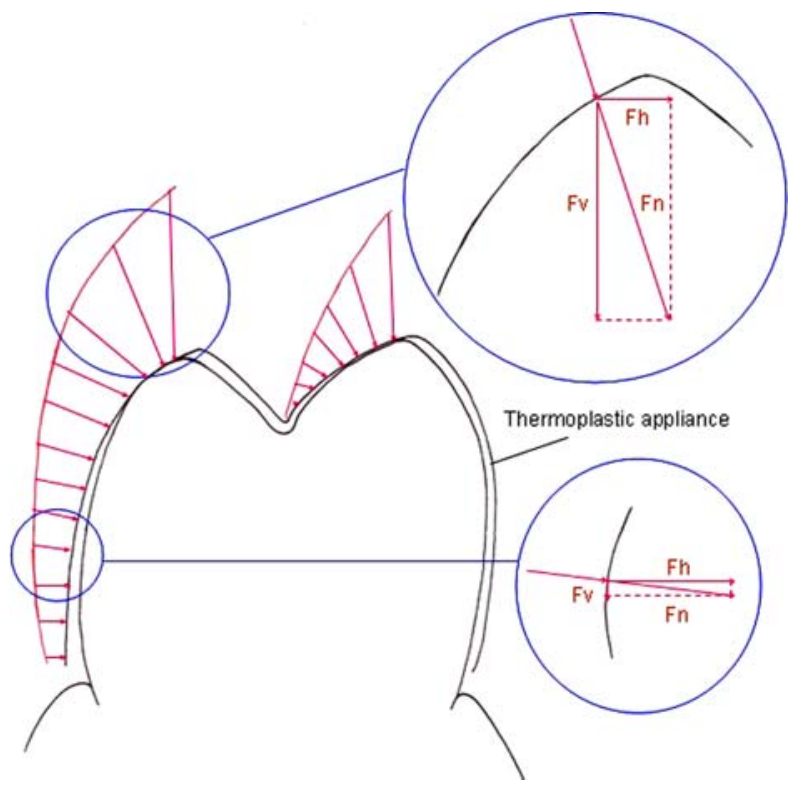

FIGURE 5. Graphic representation of the force components expected upon immediate intraoral placement of the thermoplastic appliance. The normal (or overall) force may be divided into a horizontal and a vertical component. Approaching the cusp tips the vertical component is substantial and horizontal component is comparatively small. Distant from the cusp tip the contribution of the vertical component is diminished. $F_{\mathrm{n}}=$ Normal component of the force; $F_{\mathrm{v}}=$ Vertical component of the force; $F_{h}=$ Horizontal component of the force.

of RTA wear was found to be $1.12 \mathrm{~N}(\mathrm{SE}=0.72 \mathrm{~N})$. This mean force of $1.12 \mathrm{~N}$ represents only $5 \%$ of the total force that was measured intraorally in the ClearSmile ${ }^{\circledR}$ patients. Its contribution is minimal when compared to that generated by the irrelevant pressures (Table 1), which are induced by the inherent film thickness, the appliance seating and the sliding contact between the tooth and appliance. The mal-positioned tooth is receiving the force only from RTA, as the interference in force distribution from the adjacent teeth is not possible due to the fact that the RTA is designed to hold all other teeth as anchorage to secure the displacement of the concerned tooth.

The calculated mean force of $1.12 \mathrm{~N}(\mathrm{SE}=0.72 \mathrm{~N})$ applied to the first premolar is theorized to be an average of the initial and final forces. This is based on the hypothesis that the force is reducing linearly from RTA issue to retrieval. Due to the fact that the initial force is much higher than the final one, it is reasonable to contend that the change of the force imparted by RTA does not take a linear pattern, rather, it would reduce exponentially. This force pattern implies that for the majority of the 2 week period during which the appliance is worn, the force exerted is less than $1.22 \mathrm{~N}$.

It is not surprising that the mean calculated force for the initial RTA placement is very high $(5.12 \mathrm{~N}$, $\mathrm{SE}=0.80 \mathrm{~N}$ ), as this measurement is made when the
Erkudor ${ }^{\circledR}$ thermoplastic material is at its most resilient status. Over the 2 weeks of RTA wear the function of thermoplastic material diminishes and even deteriorates, ${ }^{6}$ leading to a drastically decreased force upon RTA retrieval $(-2.67 \mathrm{~N}, \mathrm{SE}=0.69 \mathrm{~N})$.

Some studies found that the material could be modified by prolonged exposure to heat, moisture and enzymes of the oral cavity. ${ }^{4,7,16}$ A recently published study, utilizing fourier transform infrared microspectroscopic analysis of thermoplastic appliance specimens, revealed that the devices were composed of polyurethane with added methylene diphenyl diisocyanate and 1,6 hexanediol. ${ }^{16}$ Erkudor plastic, the material from which ClearSmile appliances is constructed is also composed of polyurethane. The study also assessed the properties of the material before and after 2 weeks of intraoral wear. Optical microscopic analysis revealed that following the 2 weeks of intraoral exposure, there were considerable changes in surface morphology including distortion, cracking, wear of contact points, abrasion at cusp tips and calcified proteinaceous biofilms. A Vickers hardness test of the retrieved appliances also revealed an increase in hardness of the buccal segment. ${ }^{16}$ Therefore the declined force may relate to the stiffening of the materials and loss of its elasticity or springiness.

It is suspected that the negative value occurs because the passive RTA pressure for calibrated records were conducted with a newly constructed one, but and the final RTA pressure records were obviously performed upon a thermoplastic material that had deteriorated due to exposure to 2 weeks of oral conditions. Ideally the passive pressure measurement should be performed with a passive RTA that has experienced 2 weeks of oral conditions. However, none of the patients in the study wore a passive RTA so such a measurement was not possible.

Another possible explanation on the phenomenon of a rapid force decline is that with time passage, the tooth imposed with the force moves away from the RTA acting surface, leading to a decreased force level. This explanation, however, may not be convincing as only $0.5 \mathrm{~mm}$ of tooth displacement is allowed for each RTA, a tiny position change that could hardly reduce the force so considerably.

There is expected to be variation in the pressure applied to the teeth by removable appliances including RTA, as there are no fixed contacts with the teeth to be moved in dentition. In this study, there is also variation in the pressure recording process with the Pressurex ${ }^{\circledR}$ film especially when the appliance is being inserted. This may explain the considerable variation between the three repeated pressure film measurements (Table 2). Furthermore, since during the clinical experiment there was no control between the contacting areas, it is 
possible that the dye release happened due to greater area contact in some cases and to greater force contact in others. That could also explain the large variation found in the data set.

\section{CONCLUSIONS}

(1) A novel pressure sensitive film approach - Pressurex ${ }^{\circledR}$ film was used in this study to measure the force generated by RTA against the orthodontic tooth. The average force magnitude exerted by ClearSmile ${ }^{\circledR}$ RTA to move the tooth buccally by $0.5 \mathrm{~mm}$ is $1.12 \mathrm{~N}$.

(2) The rapid diminish in magnitude from initial RTA issue to final removal indicates a exponential, but not linear, pattern of force change during the each RTA wear.

(3) In vitro force measurement with Pressure ${ }^{\circledR}$ film staining seems to be accurate when all the irrelevant force sources are excluded.

\section{REFERENCES}

${ }^{1}$ Celi, A., G. Merrill-Skoloff, P. Gross, S. Falati, D. S. Sim, R. Flaumenhaft, B. C. Furie, and B. Furie. Thrombus formation: direct real-time observation and digital analysis of thrombus assembly in a living mouse by confocal and widefield intravital microscopy. J. Thromb. Haemost. 1:6068, 2003.

${ }^{2}$ Djeu, G., C. Shelton, and A. Maganzini. Outcome assessment of Invisalign and traditional orthodontic treatment compared with the American Board of Orthodontics objective grading system. Am. J. Orthod. Dentofacial Orthop. 128:292-298, 2005: discussion 298, 2005.

${ }^{3}$ Dorow, C., N. Krstin, and F. G. Sander. Experimental model of tooth mobility in the human "in vivo". Biomed. Tech. (Berl.) 47:20-25, 2002.

${ }^{4}$ Eliades, T., G. Eliades, and D. C. Watts. Structural conformation of in vitro and in vivo aged orthodontic elastomeric modules. Eur. J. Orthod. 21:649-658, 1999.

${ }^{5}$ Hale, J. E., and T. D. Brown. Contact stress gradient detection limits of Pressensor film. J. Biomech. Eng. 114:352-357, 1992.
${ }^{6}$ Hori, K., T. Ono, H. Iwata, T. Nokubi, and I. Kumakura. Tongue pressure against hard palate during swallowing in post-stroke patients. Gerodontology 22:227-233, 2005.

${ }^{7}$ Huget, E. F., K. S. Patrick, and L. J. Nunez. Observations on the elastic behavior of a synthetic orthodontic elastomer. J. Dent. Res. 69:496-501, 1990.

${ }^{8}$ Kesling, H. D. The philosophy of the tooth positioning appliance. Am. J. Orthod. Oral Surg. 31:297-304, 1945.

${ }^{9}$ Liggins, A. B. The practical application of Fuji Prescale pressure-sensitive film. In: Optical Measurement Methods in Biomechanics, edited by J. F. Orr and J. C. Shelton. London: Chapman and Hall, 1997, pp. 173-186.

${ }^{10}$ Liggins, A. B., and J. B. Finlay. Image-averaging in the analysis of data from Fiji pressure-sensitive film. Exp. Technique 21:19-22, 1997.

${ }^{11}$ Liggins, A. B., W. R. Hardie, and J. B. Finlay. The spatial and pressure resolution of Fuji pressure-sensitive film. Exp. Mech. 35:166-173, 1995.

${ }^{12}$ Liggin, A. B., K. Surry, and J. B. Finlay. Sealing Fuji prescale pressure-sensitive film for protection against fluid damage: the effects on its response. Strain 31:57-62, 1995.

${ }^{13}$ Liu, Z. J., and S. W. Herring. Bone surface strains and internal bony pressures at the jaw joint of the miniature pig during masticatory muscle contraction. Arch Oral Biol. 45:95-112, 2000.

${ }^{14}$ Nakagami, G., H. Sanada, C. Konya, A. Kitagawa, E. Tadaka, and K. Tabata. Comparison of two pressure ulcer preventive dressings for reducing shear force on the heel. J. Wound Ostomy Continence Nurs. 33:267-272, 2006.

${ }^{15}$ Robinson, J. W. Spectrophotometry, colorimetry and polarimetry. In: Undergraduate Instrumental Analysis, edited by J. W. Robinson. New York: Marcel Dekker Inc, 1995, pp. 386-403.

${ }^{16}$ Schuster, S., G. Eliades, S. Zinelis, T. Eliades, and T. G. Bradley. Structural conformation and leaching from in vitro aged and retrieved Invisalign appliances. Am. J. Orthod. Dentofacal Orthop. 126:725-728, 2004.

${ }^{17}$ Sheridan, J. J. The Readers' Corner. 2. What percentage of your patients are being treated with Invisalign appliances? J. Clin. Orthod. 38:544-545, 2004.

${ }^{18}$ Shrive, N. G. Soft tissue strain measurement. In: Optical Measurement Methods in Biomechanics, edited by J. F. Orr and J. C. Shelton. London: Champman and Hall, 1997, pp. 154-159.

${ }^{19}$ Venugopal, K., and R. N. Saha. New, simple and validated UV-spectrophotometric methods for the estimation of gatifloxacin in bulk and formulations. Farmaco. 60: 906-912, 2005.

${ }^{20}$ Yip, S. K., M. W. Pang, and D. S. Sahota. Measurement of tension-free vaginal tape trocar insertion and exit forces. Gynecol. Obstet. Invest. 62:55-60, 2006. 